\title{
ECONOMÍA ECOLÓGICA DEL FRACKING: TENSIONES ENTRE EL AUTOABASTECIMIENTO ENERGÉTICO Y LA REPRIMARIZACIÓN DE LA ECONOMÍA EN LA ARGENTINA DEL SIGLO XXI
}

\author{
Ecological Economics of Fracking: Tensions Between Energy \\ Self-Sufficiency and Economic Re-Primarization in Argentina \\ Of the XXI Century
}

\author{
SANDRA BETTINA FERRANTE ${ }^{1}$ \\ ${ }^{1}$ Universidad Pablo de Olavide (UPO)
}

E-mail: sandra.ferrante@gmail.com

\begin{abstract}
Resumen
La extracción de hidrocarburos no convencionales, se expande rápidamente en Argentina. De esa manera se pretende avanzar hacia el autoabastecimiento de combustibles a escala nacional. La actual crisis energética en Argentina suele considerarse un problema de oferta. Los análisis de las transformaciones en la demanda derivadas de la reprimarización de la economía durante el corriente siglo son escasos y/o parciales. Tanto el neoextractivismo como el fracking resultan viables en base al predominio de análisis basados en la alta rentabilidad monetaria que involucran. Sin embargo, el conocimiento biofísico de la economía de la energía es irreductible a cuestiones crematísticas. El objetivo general de este trabajo es estudiar, desde la perspectiva de la economía ecológica, las relaciones entre la problemática del autoabastecimiento de combustibles fósiles en Argentina y las transformaciones en la demanda de esos recursos, derivadas de la expansión del neoextractivismo. Para ello, se abordan las siguientes preguntas: i) ¿cuáles han sido los cambios en la demanda de energía derivados de la expansión(a) de la agricultura industrial y (b) de la megaminería, en la Argentina del siglo XXI? ii) ¿cuáles serían las transformaciones en la disponibilidad neta de energía a partir de la expansión de la extracción de HCNC en Argentina, en comparación con la correspondiente a los homólogos tradicionales?
\end{abstract}

Palabras claves: Déficit energético, Fractura hidráulica, Reprimarización, Vaca Muerta.

\begin{abstract}
Unconventional hydrocarbons extraction is expanding in Argentina. The aim of these is to move towards self-sufficiency in fuels and to solve the current energy crisis in Argentina, often considered a supply problem. The analysis of changes in demand resulting from the reprimarization of the economy during the current century are scarce and / or partial. Both the neo-extractivism as fracking are viable based on the predominance of monetary analysis and high profitability. However, the biophysical knowledge of energy economics can not be reduced to chrematistic issues. The overall objective of this work is to study, from ecological economics perspective, relations between the problems of self-supply of fossil fuels in Argentina and the changes in the demand for these resources, resulting from the expansion of neo-extractivism. To achieve this goal, the following questions are addressed: i) What have been the changes in energy demand resulting from expansion (a) of industrial agriculture and (b) of large scale mining, in today's Argentina. Additionally, this work discuss about ii) What would be the changes in net energy availability from expanding shale extraction in Argentina, compared with the corresponding conventional counterparts?
\end{abstract}

Keywords: Energy deficit, Hydraulic fracturing, Reprimarization, Vaca Muerta. 


\section{INTRODUCCIÓN}

En Latinoamérica, los hidrocarburos constituyen la fuente energética mayoritaria y son considerados la principal riqueza natural y estratégica para la región (Mansilla 2011). En ese marco, la extracción de gas y petróleo se presenta usualmente como una oportunidad para sostener el crecimiento económico en base a recursos propios, lo cual facilitaría satisfacer las necesidades más urgentes de la población y redundaría en mayores beneficios sociales de las clases trabajadoras (García- Linera 2012). Recientemente, a los beneficios que suponen los recursos fósiles tradicionales se suman las expectativas de extracción de los hidrocarburos localizados en las formaciones no convencionales. Éstas últimas, para el caso de Argentina, han sido estimadas entre las primeras del mundo (EIA 2011).

En función de lo mencionado, la extracción de petróleo y gas no convencional (NC) en Argentina, constituye la principal apuesta gubernamental para superar el actual déficit en la balanza comercial, asociado con las importaciones de combustibles fósiles. De ahí que, la expansión de la fractura hidráulica (una de las tecnologías empleadas en la extracción de NC), también conocida como fracking, sea presentada desde el gobierno nacional como una oportunidad tanto para disponer de energía para sostener el crecimiento económico como para recuperar el autoabastecimiento energético.

Un aspecto que no suele visualizarse en las propuestas convencionales de solución al desbalance energético argentino es que: los flujos monetarios aunque podrían ser útiles para cubrir el déficit de balanza comercial, no necesariamente lo son para sustituir flujos biofísicos, como los que corresponden con la matriz energética de la economía. Dado que no es posible sustituir completamente los recursos naturales con capital (Stern 1997) y que recuperar o sostener el autoabastecimiento energético implica flujos de energía asociados con recursos naturales; el conocimiento de las dimensiones biofísicas de la economía de la energía NC constituye un problema teórico (y práctico) que debe abordarse en forma específica.

Sin embargo, el estudio de los flujos energéticos de la economía suele relegarse. En Argentina, al igual que en otros países, predominan los análisis económicos ortodoxos y de ellos deriva la focalización casi exclusiva en la dimensión monetaria. De ahí que los problemas de déficit energético se visualicen a partir del impacto negativo en las reservas de moneda extranjera asociados con las importaciones crecientes de hidrocarburos (Giuliani 2013). Por otra parte, la pérdida del autoabastecimiento de combustibles fósiles se suele asociar con una problemática de disminución de la extracción y, por lo tanto, de la oferta. En coherencia con ello, la responsabilidad por la creciente importación de combustibles fósiles suele identificarse con el rol depredador de REPSOL en la gestión de YPF (Sabbatella 2014). Los eventuales cambios en la demanda de vectores energéticos, relacionados con el proceso de reprimarización actual (Merlinsky 2013), apenas se han estudiado.

En función de lo anterior, el objetivo general de este trabajo es estudiar las relaciones entre la problemática del autoabastecimiento de combustibles fósiles en Argentina y las transformaciones en la demanda de esos recursos, derivadas de la expansión del neoextractivismo. En particular se abordan las siguientes preguntas: i) ¿cuáles han sido los cambios en la demanda de energía derivados de la expansión (a) de la agricultura industrial y (b) de la megaminería, en la Argentina del siglo XXI? ii) ¿cuáles serían las transformaciones en la disponibilidad neta de energía a partir de la expansión de la extracción de HCNC en Argentina, en comparación con la correspondiente a los homólogos tradicionales?

La perspectiva bajo la cual se desarrolla este estudio corresponde a la economía ecológica, en complemento con la ecología política (Martínez-Alier 1995). Desde ese marco conceptual transdiciplinar, tanto la producción como la transformación de energía y materiales son cuestiones centrales (Ramos-Martín 2003). A su vez, desde ese enfoque es posible visualizar que la extracción de recursos naturales, como los combustibles fósiles, aunque se contabilice como producción puede implicar un crecimiento económico depredador basado en el mayor empleo de energía y materiales (Martínez-Alier \& Roca 2001 en Vallejos 2006). Cabe aclarar que el avance que aquí se presenta es parte de una investigación en curso y reviste continuidad con trabajos anteriores de la autora, tanto en forma exclusiva como en colaboración. Al mismo tiempo, los procesos productivos y extractivos analizados no comprenden la totalidad de las transformaciones posibles de ser asociadas con la reciente reprimarización de la economía Argentina. De ahí, que para la continuación de esta investigación se considere relevante incluir el análisis de las transformaciones en el metabolismo energético relativas al sector ganadero y al denominado complejo oleaginoso.

\section{MATERIALES Y MÉTODOS}

La metodología empleada involucra el análisis de fuentes secundarias y el procesamiento de información cualicuantitativa relativa a los flujos de energía de la economía argentina contemporánea. En relación con esto último, cabe aclarar que el recorte temporal abarca los años transcurridos durante el corriente siglo. Numerosos autores han analizado las características del proceso de expansión del 
neoextractivismo y la reprimarización de la economía argentina. Aun así, es difícil establecer algún consenso sobre qué se entiende por reprimarización (Slipak 2013). Aquí usaremos indistintamente los términos reprimarización y neoextractivismo. Las actividades que analizaremos aquí, en términos de demanda energética, serán la expansión del agronegocio, relacionado con la agricultura industrial extensiva de gran escala y la megaminería.

Las transformaciones en la demanda de combustibles fósiles en relación con la expansión de la agricultura industrial, se estudian a escala nacional y en relación con los principales cultivos de granos. En cambio, para el caso de la megaminería se consideran los emprendimientos mineros metalíferos de mayor envergadura, en la Argentina del corriente siglo. El estudio biofísico de la extracción de hidrocarburos no convencionales y el fracking, se refiere a las formaciones geológicas Vaca Muerta y Los Molles, localizadas en la provincia de Neuquén.

Las transformaciones en la disponibilidad energética se refieren a la rentabilidad biofísica, es decir a la relación entre "salidas" ("output") y "entradas" ("input") aplicada, en este caso, a procesos de extracción de HCNC. Para analizar esa relación considerando la "ganancia" neta de energía, se han desarrollado un conjunto de índices (Murphy \& Hall 2010). Entre ellos, uno de los más difundidos es la Tasa de Retorno Energético (TRE) o EROI (por la sigla en inglés de "Energy Return on Energy Investment"). El EROI se define como el cociente entre la cantidad de energía obtenida en un determinado proceso de extracción, o "producción" de energía, y la cantidad de energía empleada para ello (Cleveland et al 1984, Murphy \& Hall 2010, Murphy et al. 2011).

Un aspecto a considerar en relación con las estimaciones de rendimiento energético es que el EROI (o la TRE) es un índice que agrega información y que ello resulta en limitaciones que aquí no consideraremos. Aún así, dado lo preliminar de este trabajo usaremos el índice mencionado para estimar la disponibilidad de energía neta de los HCNC y posteriormente comparar esa relación con la correspondiente a los fósiles tradicionales. El EROI que aquí se estima corresponde principalmente al upstream y los primeros eslabones del downstream. Para estimar la cantidad de energía empleada en la extracción NC, se consideran flujos directos e indirectos.

El recorte del análisis en relación a la expansión del fracking se justifica fácilmente dado que en otras posibles zonas de extracción de HCNC el avance de esa tecnología aún es insignificante. Sin embargo, dado que no ocurre lo mismo, en la selección de las actividades seleccionadas como claves en el proceso de reprimarización reciente, se justifica brevemente la acotación elegida. En primer lugar, insistir en que se trata de una investigación en curso. En segundo, mencionar que tanto la expansión sojera como la de la megaminería involucran cambios de orden de magnitud en el uso de recursos y energía fósil, en lapsos temporales relativamente cortos. En cambio, en sectores como la ganadería las tendencias podrían incluir información contradictoria. Ejemplo de ello es la cuestión de la intensificación de la ganadería que convive con un desplazamiento de la actividad hacia áreas marginales (Charvay 2012). El feedlot (o engorde a corral) requiere más energía fósil por kilogramo de carne producida que los sistemas extensivos pero los sistemas extensivos en áreas marginales posiblemente requieran menos energía fósil que los que incluyen pasturas cultivadas. La ganadería intensiva incrementó notoriamente su peso relativo en el total de animales faenados pero, al mismo tiempo, se ha desplegado un proceso de reducción del stock ganadero (Charvay 2012).

En futuros trabajos, profundizaremos el análisis aquí planteado incorporando tanto las eventuales transformaciones en la actividad pecuaria como los consumos energéticos relativos a la industria oleaginosa. En relación con esta última actividad, sólo mencionar dos cuestiones que llevaron a excluirla del recorte analítico de este trabajo. Por un lado, la complejidad de la contabilidad energética de una actividad industrial que involucra diferentes vectores energéticos (Giampietro \& Sorman 2010). Por otro, la discusión sobre si es parte de la reprimarización reciente $o$, por el contrario, de una eventual (re)industrialización (Frechero 2013).

\section{RESULTADOS}

\section{Cambios en la demanda de energía, en la Argentina del siglo XXI}

Hasta hace aproximadamente una década atrás, la crisis energética argentina parecía no relacionarse con transformaciones en la demanda de combustibles sino con estrategias empresariales para aumentar la rentabilidad (Kozulj 2005). Más recientemente, las explicaciones para el actual déficit energético involucran sobre-explotación de los yacimientos y exportación de recursos, por parte de empresas extranjeras (Sabbatella 2014). En cambio, las transformaciones en la demanda de energía fósil en la Argentina actual constituyen un aspecto poco estudiado en asociación con la crisis energética del corriente siglo.

Un estudio realizado en base a encuestas industriales en cuatro países latinoamericanos permite concluir que recientemente "la manifiesta especialización de la región en sectores intensivos en recursos naturales ha contribuido a un 
patrón de alto consumo energético" (Altomonte et al. 2011). Aunque Argentina no estaba incluida en el análisis mencionado, todo indica que su situación no es excepcional. Para el período 2004-2014, el consumo de energía fósil en Argentina ha pasado de 425 a 662 miles de barriles diarios de petróleo y de 37.9 a 47.2 billones de metros cúbicos de gas natural (BP 2015). De éstos últimos, 6.5 billones fueron importados (BP 2015).

Más allá de los debates acerca de la reprimarización de la economía argentina y del auge del extractivismo, es evidente que actividades como la minería a gran escala y la agricultura industrial extensiva se han expandido notoriamente a lo largo de la década reciente pasada. Algunos indicadores de la presencia creciente de las actividades mencionadas son: i) el crecimiento acumulado del valor de las exportaciones mineras entre 2002 y 2011, alcanzó un 434\% (Secretaría de Minería 2012), ii) el número de proyectos mineros, para el mismo período, aumentó en un 3 311\% (Secretaría de Minería 2012), iii) la producción de granos pasó de 69.7 millones de toneladas en 2003/04 a 110.7 millones de toneladas durante 2013/14 (Calzada et al 2015), iv) el área sembrada con cereales, oleaginosas y otros cultivos industriales pasó de 28.8 a 36.5 millones de hectáreas para el período mencionado en (iii) (Calzada et al 2015).

En las dos sub-secciones que comprenden este apartado, se sistematiza información sobre la evolución del consumo de combustibles fósiles vinculada con la expansión de la agricultura industrial extensiva y la minería a gran escala.

\section{Demanda ligada a la expansión de la agricultura industrial}

En lo que va de siglo, la superficie cultivada en Argentina aumentó cerca de diez millones de hectáreas. Los cultivos con los que se asocia tal incremento son principalmente, soja y maíz. En cambio, la superficie sembrada con cereales permaneció relativamente estable. La frontera agrícola se expandió cambiando el uso de tierras antes marginales, destinadas a ganadería y/o forestales. De ahí, la asociación con mayores consumos de combustibles fósiles, utilizados directa e indirectamente.

Desde comienzos de los noventa, la superficie sembrada con soja aumentó alrededor de cuatro veces, alcanzando el corriente año alrededor de 20 millones de hectáreas (has). Esta última cifra es casi el doble del área sembrada a principios de los 2000. Para el caso del maíz, el incremento es similar, dado que pasó de un área de siembra de 3.6 millones de has en la campaña 2000/2001 a unas 6 millones durante 2013/2014.

\footnotetext{
${ }^{1}$ Según la Secretaría de Energía (2014) durante el 2012 se importaron 2260
}

El consumo directo de gas oil asociado con el cultivo de soja, incluyendo las actividades de siembra, fertilización, cosecha, acondicionamiento y transporte, se estima en 62.6 litros por ha (Roulet 2013). De ahí que para la campaña 2013/14, en sólo un cultivo se habrían utilizado 1252 millones de litros de gas oil (1.25 millones de $\mathrm{m}^{3}$ ). Ese volumen de combustible equivale al $47 \%$ del gasoil importado por Argentina durante el año $2012^{1}$ y es del mismo orden de magnitud que el total del consumo de gas oil para la campaña 2007/08 (Donato 2009).

Para el caso del maíz, la utilización directa de gas oil es de unos 120.9 litros/ha y para el girasol alcanzaría unos 58 litros/ha (Roulet 2013). Contabilizando esos consumos se agregarían 725.4 millones de litros (para las 6 millones de has sembradas con maíz) y 75.4 millones de litros (para las 1.3 millones de has sembradas con girasol). Adicionalmente, utilizando el mismo consumo por ha que el estimado por Roulet (2013) para girasol, la producción de trigo involucraría unos 214 millones de litros, para unas 3.7 millones de has. En todos los casos, las estimaciones consideran $300 \mathrm{~km}$ para los fletes largos y utilizan una simplificación de labores, sin discriminar siembra convencional o directa (Roulet 2013).

Entre la campaña agrícola 2011/12 y la siguiente, el consumo directo de combustible, en el sector, aumentó un $6.8 \%$; aproximadamente la mitad del consumo corresponde al transporte de granos (Calzada \& Mateo 2012), razón por la cual en las estadísticas habituales podría registrarse incorrectamente. De hecho, el único trabajo publicado sobre consumo directo de gas oil en el sector agropecuario argentino, para la campaña 2007/2008, sólo incluye los traslados dentro de la explotación agropecuaria (Donato 2009). El promedio simple de la incidencia de los fletes largos en las estimaciones de consumo de combustible por cultivo, proporcionadas por Roulet (2013), corresponde con un $49 \%$ del total.

Las "medidas de protección de cultivos" engloban tanto prácticas de labranza como aplicación de diversos agrotóxicos. En el primer caso, labranza, estas medidas implican mayoritariamente consumos directos de combustible. En el segundo, además de los consumos directos, es importante la incidencia del costo energético, indirecto, de fabricación de los productos a aplicar. En ambos casos, un análisis más detallado incluiría la utilización indirecta de energía asociada con las maquinarias y equipos agrícolas. Solamente en la aplicación se emplea alrededor del $10 \%$ del volumen de gas oil contabilizado por hectárea (Roulet 2013). Esa cifra es muy inferior a la correspondiente con la utilización indirecta de energía asociada con su fabricación, envasado y transporte. En Argentina, la

miles de toneladas equivalentes de petróleo (kTEP), lo cual equivale a 2,6 millones de $\mathrm{m}^{3}$ de gas oil. 
utilización de agroquímicos aumenta considerablemente y en forma sostenida desde principios del corriente siglo. Entre 1990 y 2012, tanto en volúmenes como en facturación, el mercado de herbicidas se incrementó más de $1000 \%$ (Moltoni \& Duro 2014). La mayor parte de los fitosanitarios aplicados son herbicidas (59\%) y se asocian con cultivos en sistemas de siembra directa o labranza cero. Cerca de la mitad de los más de 245000 toneladas de herbicidas comercializadas en Argentina durante 2012, se utilizaron para producir soja (Moltoni \& Duro 2014).

El herbicida de mayor difusión en Argentina es el glifosato, por eso lo utilizaremos aquí como ejemplo y primera aproximación a la problemática de contabilizar los costos energéticos del sector agropecuario. El consumo de glifosato para la campaña 2012/13 fue de aproximadamente 200 millones de kg (Consejo Científico Interdisciplinario 2009). El costo energético de sintetizar esa molécula es de alrededor de 511MJ por cada kg producido (Green 1987). Para el actual nivel de consumo en Argentina, ello implicaría unos 102200 millones de MJ anuales, que equivaldrían a 2.6 millones de $\mathrm{m}^{3}$ de gas oil ${ }^{2}$. Ese volumen de combustible, es similar al total de gas oil importado durante 2012 a nivel nacional. Algo más de la mitad del glifosato utilizado se produce en Argentina, con lo cual el costo físico de la actividad incide en forma directa en la demanda energética nacional.

En cuanto a los fertilizantes utilizados en la agricultura industrial, es importante notar que todos los nitrogenados (ej. urea, nitrato de amonio) se producen a partir de gas natural (y aire). En este caso, los combustibles fósiles no sólo se requieren como vectores energéticos sino que adicionalmente se utilizan como materia prima para la elaboración de los productos. En posteriores análisis, correspondería incluir tales consumos energéticos. Según la Cámara de la Industria Argentina de Fertilizantes y Agroquímicos (2014), durante la campaña agrícola 2012/13 se utilizaron más de 3.2 millones de toneladas de fertilizantes, la mitad de los cuáles se produjo en Argentina.

\section{Demanda ligada a la expansión de la megaminería en Argentina}

En Argentina, la información sobre proyectos mineros es escasa y opaca, incluso cuando proviene de organismos oficiales. Emprendimientos mineros que están en producción desde hace varios años, son listados en el sitio web de la Secretaría de Minería de la Nación (ver sección de MineríaProyectos y Prospectos), indicando que están en prospección.

\footnotetext{
2 Se utilizan conversiones sin considerar los vectores energéticos utilizados, sólo como aproximación preliminar.

${ }^{3}$ Esta cifra coincide con lo informado para grandes usuarios del mercado
}

El país no dispone de relevamientos sistemáticos sobre consumos de energía por parte de la industria minera. Tal situación, contrasta por un lado, con la crítica situación energética en Argentina. Y, por otro, con la evidencia de que en años recientes, se estaría intensificando el uso de energía en la minería a gran escala. Ejemplo de ello, la minería chilena, aumentó alrededor del 10\% la extracción de cobre incrementando en casi un $60 \%$ la utilización de energía asociada (De-Solminihac 2013).

En esta sub-sección, se presenta información sistematizada sobre los emprendimientos mineros de gran escala, en operación en Argentina desde el corriente siglo. Se entiende por gran escala, aquellas mineras con capacidad para procesar diariamente más de 2000 toneladas de materiales. Aun cuando los datos disponibles fueran diarios, en la Tabla 1 y con el objetivo de facilitar las comparaciones, se presenta la información en base anual. Es importante mencionar que la capacidad de procesamiento es dinámica. Cuando las empresas mineras deciden bajar la ley de corta y/o aumentar la vida útil de los emprendimientos, suelen realizar algún tipo de inversión para aumentar la capacidad de trabajo. En función del consumo de energía eléctrica, el mayor proyecto de extracción de metales en Argentina es Bajo La Alumbrera. En el informe de 2014, la empresa minera que lo opera, indica un consumo directo de electricidad de $847376 \mathrm{MWh}^{3}$. Según el mismo informe, un $44 \%$ de esa electricidad es obtenida en plantas que utilizan gas natural. Aunque la empresa informa que uno de sus principales insumos son combustibles y lubricantes, no indican qué cantidades de éstos utilizan. La cantidad de electricidad informada equivalía a la cuarta parte del total distribuido en el noroeste argentino y al $87 \%$ del consumo total en la provincia de Catamarca (Coria 2007).

Para el caso de Barrick Gold, el consumo de energía correspondiente a sus actividades en Argentina, superó en 2012 los 8765000 GJ (Barrick Argentina 2013), cifra que duplica el consumo informado para el año 2010 (Barrick Argentina 2011). Realizando una conversión directa (sin considerar las transformaciones ni vectores energéticos, ya que no se dispone de información desagregada), tal utilización de energía resultaría equivalente a $251815 \mathrm{~m}^{3}$ de gas oil. Ese volumen de combustible, utilizado por una sola empresa, supera el $12 \%$ de la cantidad de gas oil importado a nivel nacional durante 2011.

mayorista en la provincia de Catamarca. Por lo tanto, es probable que excluya los consumos de la empresa en las provincias de Tucumán y Santa Fe. 
Tabla 1. Grandes proyectos mineros en operación en Argentina

\begin{tabular}{|c|c|c|c|c|c|c|}
\hline Lugar & Provincia & Minerales & Empresa & $\begin{array}{c}\text { Capacidad de } \\
\text { procesamiento } \\
\text { (toneladas/año) }\end{array}$ & $\begin{array}{l}\text { Extracción final } \\
\text { (kg/año) }\end{array}$ & $\begin{array}{l}\text { Ley de corta } \\
\text { (g/ton) }\end{array}$ \\
\hline Bajo la Alumbrera & Catamarca & $\begin{array}{l}\text { cobre, oro, plata } \\
\text { y molibdeno }\end{array}$ & $\begin{array}{l}\text { Minera } \\
\text { Alumbrera LTD }\end{array}$ & 43800000 & $\begin{array}{c}17000 \mathrm{Au} \\
180000.000 \mathrm{Cu} \\
468000 \mathrm{Mb}\end{array}$ & $\begin{array}{c}1 \mathrm{Au} \\
0.76 \% \mathrm{Cu}\end{array}$ \\
\hline Cerro Vanguardia & Santa Cruz & oro y plata & $\begin{array}{l}\text { Cerro } \\
\text { Vanguardia S.A. } \\
\text { (Anglo Gold } \\
\text { Ashanti) }\end{array}$ & 1095000 & $\begin{array}{c}5925 \mathrm{Au} \\
79378 \mathrm{Ag}\end{array}$ & $\begin{array}{l}8.9 \mathrm{Au} \\
110 \mathrm{Ag}\end{array}$ \\
\hline Pirquitas & Jujuy & plata, zinc & Silver Standar & 2190000 & $246640 \mathrm{Ag}$ & $\begin{array}{c}189 \mathrm{Ag} \\
0.61 \% \mathrm{Zn}\end{array}$ \\
\hline Veladero & San Juan & oro y plata & Barrick Gold & 13140000 & $21885 \mathrm{Au}$ & $(1.7 \mathrm{Au})$ \\
\hline Gualcamayo & San Juan & $\begin{array}{l}\text { oro, plata y } \\
\text { cobre }\end{array}$ & Yamana Gold & 44000000 & $5115 \mathrm{Au}$ & $0.6 \mathrm{Au}$ \\
\hline Manantial Espejo & Santa Cruz & oro y plata & $\begin{array}{l}\text { Minera Triton / } \\
\text { Pan American } \\
\text { Silver }\end{array}$ & 720000 & $\begin{array}{c}87883 \mathrm{Ag} \\
1723 \mathrm{Au}\end{array}$ & $(2.4 \mathrm{Au})$ \\
\hline Sierra Grande ${ }^{* *}$ & Río Negro & hierro & $\begin{array}{l}\text { China } \\
\text { Metalurgical } \\
\text { Group }\end{array}$ & $\mathrm{N} / \mathrm{D}$ & $2800000 \mathrm{Fe}$ & $68.55 \mathrm{Fe}$ \\
\hline Total de oro extraído & & & & & 51648 & \\
\hline Total de plata extraída & & & & & 413901 & \\
\hline \multicolumn{7}{|c|}{ Proyectos paralizados o en construcción } \\
\hline Pascua-Lama & $\begin{array}{l}\text { San Juan (y } \\
\text { Chile) }\end{array}$ & oro y plata & Barrick Gold & 12045000 & $\begin{array}{l}22679 \mathrm{Au}^{*} \\
992233 \mathrm{Ag}^{*}\end{array}$ & $\mathrm{~N} / \mathrm{D}$ \\
\hline Agua Rica & Catamarca & $\begin{array}{l}\text { cobre, oro, plata } \\
\text { y molibdeno }\end{array}$ & Yamana Gold & $17000000^{*}$ & $\begin{array}{c}3900 \mathrm{Au}^{*} \\
150000000 \mathrm{Cu}\end{array}$ & $\begin{array}{c}0.23 \mathrm{~g} / \mathrm{t} \mathrm{Au} \\
0.62 \% \mathrm{Cu}\end{array}$ \\
\hline Pachon & San Juan & $\begin{array}{l}\text { cobre y } \\
\text { molibdeno }\end{array}$ & Xstrata Copper & 58400000 & $280000000 \mathrm{Cu}$ & $0.2 \% \mathrm{Cu}$ \\
\hline
\end{tabular}

Fuentes: Elaboración propia a partir de datos disponibles en http://www1.hcdn.gov.ar/dependencias/cmineria.htm y http://www.segemar.gov.ar/bibliotecaintemin/FICHASTECNICASMINERAS. Los datos de extracción final corresponden al último año informado (entre 2011 y 2014). El * indica que se trata de extracción y procesamiento de materiales estimados. ${ }^{* *}$ Se presentan datos de este emprendimiento de extracción de hierro dado que se ha reactivado recientemente, aun así la actividad está declinando la extracción y la mayor ley del hierro indicaría menor consumo en la etapa de extracción. Los paréntesis indican ley de corta inferida de los datos de extracción y procesamiento.

Pan American Silver utiliza actualmente unos $18000 \mathrm{~m}^{3}$ de gas oil anuales para extraer plata en Manantial Espejo. Al mismo tiempo, desarrolla el proyecto Navidad, que implicaría procesar una cantidad de materiales siete veces mayor, y estudia la factibilidad del proyecto Calcatreu, que podría involucrar unas 8 mega-toneladas de recursos minerales (entre tres y cuatro veces el tamaño de las reservas probadas en Manantial Espejo). El caso de Manantial Espejo 
implicaría una utilización de más de 200 litros de gas oil por $\mathrm{kg}$ de plata extraída. Si tal intensidad fuera extrapolable a los demás proyectos, la extracción de plata a gran escala requeriría $88460 \mathrm{~m}^{3}$ de combustible.

A lo largo de esta investigación, se constató que no es habitual que las empresas mineras publiquen información completa y desagregada sobre consumos de electricidad y combustibles, al menos en lo relativos a sus proyectos en Argentina. ${ }^{4}$ En función de ello, se decidió estimar la utilización de energía en base a la cantidad de metal extraído. Como aproximación, se refiere la minería aurífera, ya que los grandes proyectos mineros que lo tienen como objetivo son característicos del reciente extractivismo y explican cerca del $90 \%$ del total de la extracción de oro en Argentina.

La intensidad energética por unidad de metal extraído varía en función de las características de los yacimientos y de la ley de corta. Por regla general, a mayor ley de metal menor requerimiento energético para su extracción. En la Tabla 1, se puede observar que la ley de corta varía para cada yacimiento. Aquí intentaremos dos aproximaciones. En el primer caso, usando una intensidad promedio de $143 \mathrm{GJ} / \mathrm{kg}$ de oro (Mudd 2007), la utilización anual de energía asociada con la extracción de oro en Argentina, se estimaría en 7385664 GJ. Dado que esta cifra es menor que la reportada para la operación de Veladero y que en ese yacimiento la concentración de oro es intermedia, se considera ese proyecto como referencia. Luego de ello, se obtiene una segunda estimación de 20685159 GJ.

Argentina no es un país tradicionalmente minero; es un territorio en el que la actividad se expande en condiciones de bajas leyes de mineral y menor accesibilidad (sea por el aislamiento o las profundidades). De ahí la intervención de grandes superficies y volúmenes así como el procesamiento y transporte de enormes cantidades de materiales, a través de grandes distancias. Es evidente que todo ello resulta en consumos de crecientes cantidades de energía para disponer de una misma cantidad de metal. Aun así, desde instancias gubernamentales tanto nacionales como provinciales se propone la expansión de la actividad durante los próximos años.

\section{Hidrocarburos no convencionales y disponibilidad neta de energía}

La extracción de HCNC en Neuquén es propuesta desde instancias oficiales como estrategia para incrementar la disponibilidad de energía a escala nacional y recuperar el

\footnotetext{
4 Una razón para ello es que parte de las actividades suelen subcontratarse, especialmente durante la construcción de la mina. Sin embargo, Mudd (2010) destaca la misma falta de datos para Australia.
}

autoabastecimiento de combustibles fósiles. En las expectativas asociadas con la expansión de la actividad se confunden variables monetarias y físicas. Aunque la viabilidad en base a la rentabilidad monetaria está claramente establecida, no es posible afirmar lo mismo focalizando en la rentabilidad energética. De momento, no existen estudios detallados sobre disponibilidad neta de energía asociada a la extracción de energía no convencional en Argentina. Al igual que con la industria minera, existe un notorio déficit de datos en relación con las actividades de las empresas petroleras. Ello dificulta estimar tanto la inversión energética como la cantidad de energía disponible a nivel de pozo.

Inversión energética asociada con la extracción no convencional en Neuquén

La inversión energética, sea que se extraigan HCNC o "tradicionales", es la energía que se requiere utilizar para acceder y poner a disposición los recursos fósiles. Esa energía está directamente relacionada con las tecnologías empleadas en el proceso extractivo. Una diferencia entre los hidrocarburos convencionales y los que no lo son, es el conjunto de tecnologías de extracción. La fractura hidráulica o fracking hace parte de un paquete tecnológico que se utiliza de forma generalizada para extraer gas o petróleo de reservorios no convencionales, como los shale incluidos en la formación Vaca Muerta (Neuquén). En los reservorios shale de Vaca Muerta están operando 337 pozos, a los cuáles se agregan unos 13 a 14 mensualmente (Gutierrez-Schmidt \& Alonso 2015). Aunque la apuesta por la extracción no convencional es muy reciente, actualmente incide en un 19.4 $\%$ de la producción total de la provincia de Neuquén.

La inversión energética para la explotación de los pozos shale incluye instalaciones, maquinarias e insumos que implican costos tanto directos como indirectos. Para facilitar el análisis se identificaron actividades en tres etapas: construcción, operación y distribución. Aquí se estima la inversión energética para el upstream, lo cual comprende las dos primeras etapas mencionadas y el primer tramo del downstream, lo cual incluye las pérdidas del sistema y la compresión de gas en boca de pozo. En la Tabla 2, se presentan algunos de los componentes identificados, señalando en color gris aquellos incluidos en la estimación.

La cantidad de días de perforación varía entre 30 a 45 días (datos para Loma La Lata en Chiappussi 2013), con un consumo diario de gas oil se aproxima a los 2046 litros/día (Sell et al. 2011). En función de ello, para perforación de un 
pozo horizontal se utilizan entre 61380 y 92070 litros de gas oil $^{5}$. Para el transporte de equipos se utiliza la estimación de Aucott \& Melillo (2013), lo cual supondría unos 26786 litros de gas oil adicionales por cada pozo. El traslado de personal no está contabilizado.

A ello se sumaría la cantidad de gas oil necesaria para transportar los 15000 metros cúbicos de agua a través de 50 $\mathrm{km}$, empleando camiones cisterna de 40 toneladas ${ }^{6}$. Considerando 375 viajes y un consumo de combustible de 32 litros por cada $100 \mathrm{~km}$ (Marchese \& Golato 2011), se agregarían unos 6000 litros de gas oil para el traslado de agua. A esas estimaciones, se agregaría el combustible requerido para trasladar unas 750 toneladas de arena y aditivos de fractura mediante unos 50 camiones desde el puerto de Buenos Aires ${ }^{7}$.

Entre un 15 y un $85 \%$ del fluido de fractura (agua, arena y otros aditivos) luego de inyectado, retorna a la superficie. Las diferentes alternativas de tratamiento, reciclaje $\mathrm{y} / \mathrm{o}$ disposición final en pozos sumidero (Decreto 1483/12) implican diferentes costos tanto monetarios como energéticos. Si en Vaca Muerta, la cantidad de energía empleada para tratamiento y disposición de fluidos de retorno fuera similar a la registrada para Marcellus, ello supondría unos 82000 litros de gas oil, adicionales por cada pozo (Aucott \& Melillo 2013). Esta aproximación parece razonable en función de que se estima emplear volúmenes de agua similares. Sin embargo, podrá ajustarse cuando se conozcan los sitios de disposición final, el tipo de tratamiento de depuración empleado en Neuquén y se establezca evidencia acerca de si el límite de uso de agua que fija el Decreto 1483/12 se corresponde con la realidad o no.

La incertidumbre en la información disponible persiste también en relación con los cálculos de energía relativa a usos indirectos. En este trabajo, se utiliza nuevamente la referencia de Marcellus (Aucott \& Melillo 2013), incorporando un factor de corrección de 1.45 en función de la diferencias de profundidad media. La energía estimada para contabilizar el acero, cemento y otros materiales empleados para tuberías y revestimientos se multiplicó por ese factor; para los demás rubros se utilizó el mismo dato. De esa manera se estimó una

\footnotetext{
${ }^{5}$ La cantidad de días de perforación promedio podría ser menor pero en función de la información disponible no está claro si es por la mayor incidencia de pozos verticales o por algún tipo de mejora en la implementación de la tecnología.

${ }^{6}$ Ese es el volumen indicado en el Decreto provincial. En informes de empresas que operan en la zona se mencionan cantidades hasta cuatro veces mayores (Shiratori 2012).

${ }^{7}$ Actualmente, la arena utilizada proviene de Brasil, China y EEUU.

8 Datos relativamente similares se encuentran en Clark, C., Han, J., Burnham, A., Dunn, J., \& Wang, M. (2011). Life-Cicle Analysis of Shale gas and natural gas (pp. 1-52) y Stamford, L., \& Azapagic, A. (2014). Life cycle environmental impacts of UK shale gas. Applied Energy, 134, 506-518. doi:10.1016/j.apenergy.2014.08.063
}

inversión de energía, que varió según las alternativas de perforación entre 38000 y 58000 GJ (Ferrante \& Guiliani 2014).

\section{Retorno energético del shale gas en Neuquén}

Para estimar el retorno energético, además de contabilizar la energía invertida, se requiere estimar cuánta energía se obtendría. En cuanto a la cantidad de petróleo y gas que podría obtenerse de Vaca Muerta, la información disponible aún es escasa, incierta y poco consistente. Es importante destacar que la productividad de los pozos convencionales en Argentina es relativamente baja. Según un especialista de la Fundación Bariloche, para extraer la misma cantidad de petróleo que en un pozo de Arabia Saudita, se necesitarían cincuenta pozos argentinos (Bravo 2013).

Di Sbroiavacca (2013) propone que de cada pozo de shale gas en Neuquén se extraerían unos 124 millones de $\mathrm{m}^{3}$ (EUR o estimated ultimate recovery). En cambio, el USGS (2012) estima que por cada pozo de gas $\mathrm{NC}$, se recuperan en promedio, unos 74 millones de $\mathrm{m}^{3}$ (2 $617 \mathrm{Bcf}$ ) en Haynesville, cerca de 33 millones de $\mathrm{m}^{3}$ (1 $158 \mathrm{Bcf}$ ) en Marcellus y unos 13 millones de $\mathrm{m}^{3}$ (0.470 Bcf) en Fayetesville ${ }^{8}$. En un análisis reciente (Gutierrez-Schmidt 2014) se estima el EUR para Vaca Muerta, en 82 millones de $\mathrm{m}^{3}$ o 2.8 Bcf $^{9}$ de gas (a 30 años) y $23100 \mathrm{~m} 3$ de petróleo (en pozos verticales, a 25 años) ${ }^{10}$.

A partir de las cifras mencionadas se estimaron las pérdidas, relevantes especialmente para gas natural ${ }^{11} \mathrm{y}$ el gasto energético del procesamiento en boca de pozo. Aunque las emisiones de gas son sistemáticamente negadas por la industria, tales pérdidas alcanzan entre el 1 y el 30\% del HC (Taillant et al 2013). En este trabajo se contabiliza un $8 \%$ de la energía, destinado a comprimir y procesar el gas natural en boca de pozo (EIA 2011). La conversión de gas natural y de petróleo en unidades de energía se realiza según tablas de la EIA (2014).

Si el desempeño de los pozos de shale en Vaca Muerta se correspondiera con el estimado localmente, se recuperarían 2954156 GJ en gas y 1036564 GJ de petróleo. En un trabajo reciente, se presenta una primera aproximación para el retorno energético del shale gas en 50:1 en parte ${ }^{12}$ del upstream

\footnotetext{
${ }^{9}$ Bcf indica billones de pies cúbicos. Si el EUR indicado en el estudio referenciado fuese correcto, el escenario de autoabastecimiento en base a lo estimado por Di Sbroiavacca (2013) podría pasar de 1500 a 2250 pozos anuales.

${ }^{10}$ En un reportaje reciente, J. Sapag, indicó que los pozos NC tendrían un horizonte productivo de entre 15 y 18 años (El Inversor energético \& minero, 2013).

${ }^{11}$ Los escapes de gas ocurren en toda la cadena de explotación de HC (extracción, transporte y distribución), e incluyen fracciones gaseosas disueltas en el petróleo.

12 No se incluye el costo energético de la prospección.
} 
Ambiente y Sostenibilidad 2015 (5): 97-109

Revista del Doctorado Interinstitucional en Ciencias Ambientales

ISSN: 2339-3122

Digital

(Ferrante \& Guiliani 2014) pero sin considerar las pérdidas de energía por emisiones de gas. En ese caso, se utiliza la misma metodología aplicada por Aucott \& Melillo para Marcellus (2013). A partir de las consideraciones señaladas por Yaritani \& Matushima (2014), se incorporan en la estimación anterior la energía utilizada para comprimir el gas natural (boca de pozo) y la correspondiente a las pérdidas y fugas involuntarias (con lo cual se avanza en el primer tramo del downstream). En ese caso, para del gas natural el retorno energético rondaría una relación de 5:1 y para el petróleo se

acercaría a los 13:11. La industria petrolera insiste en que las pérdidas de gas no constituyen un costo (salvo el de oportunidad). Sin embargo, además de su impacto en la disminución de la rentabilidad energética, las fugas de gas constituyen principalmente emisiones de metano. Ello determina que la huella ecológica de la extracción de gas no convencional sea mayor no sólo respecto de los HC convencionales sino incluso que la del carbón (Howarth et al. 2011)

Tabla 2. Componentes del flujo energético asociado con el shale en Neuquén.

\section{Construcción}

Operación

Distribución

Traslado de trabajadores, equipos e insumos.

Prospección-Exploración de la formación de roca madre (mapeo del área, análisis de carbono orgánico total y reflectancia, etc.).
Tratamiento del fluido de Construcción retorno, traslado, disposición del mismo y eventual reutilización. mantenimiento

corrosión. de gasoductos, y evaluación de

Perforación vertical y horizontal, construcción del pozo, cementación, etc.

Actividades de monitoreo, Distribución detección de microsísmica, supervisión del reservorio.

Traslado de insumos de fractura (ej. agua, arena, aditivos, Tratamientos, compresión. etc.)

Terminación, punzado

Mantenimiento de equipos

Comercialización

Envasado

Fracturación hidráulica, estimulación ácida

Pérdidas, venteos, quemas, fugas involuntarias.

Las relaciones estimadas, indican que por cada barril de petróleo invertido se dispondrían de unos 13 y que por cada $\mathrm{m}^{3}$ de gas podrán utilizarse 5 veces ese volumen. El menor rendimiento energético de la extracción de HCNC en comparación con los fósiles convencionales, resulta de la mayor inversión energética a realizar para obtener una misma cantidad de energía y de la menor disponibilidad energética por pozo.

La relación de energía neta (o EROI) para los pozos convencionales explotados en EE UU en 1919 era de 1000:1 y, al igual que lo estimado para Vaca Muerta, ha caído a 5:1 en años recientes (Hall et al. 2014). Un aspecto importante, relativo a la disminución del EROI, está relacionado con la

\footnotetext{
${ }^{1}$ Las pérdidas de energía en términos de fugas de gas se contabilizaron en un $10 \%$ en el primer caso. Para el caso del petróleo se estimaron escapes equivalentes al $2 \%$ de la energía a extraer. Se trata de estimaciones
}

estimación de la duración de las reservas. Éstas, suelen referirse en una cantidad de años durante los cuales estaría garantizado el abastecimiento, asumiendo tasas de crecimiento de la demanda en función de trayectorias previas. Sin embargo, dado que tales tasas de crecimiento de la demanda no se ajustan según pérdida de rentabilidad energética, las previsiones serían inferiores a las esperadas. Una cuestión derivada de la reducción de la rentabilidad energética es el impacto en el nivel de emisiones de gases contaminantes y con efecto invernadero. Menor EROI indica mayores emisiones para una misma cantidad de energía útil.

Es importante destacar que la inversión energética que aquí se calcula podría subestimar la utilización de energía relativa

conservadoras, especialmente para el gas. En una investigación aún no publicada (Schneising et al. 2014) se determinan pérdidas de gas (en masa y en energía) para diferentes regiones de EEUU, evidenciando que los escapes de metano se están subestimando en forma sistemática. 
a la extracción de HCNC. Ello es así, porque entre otros factores no se consideraron actividades que exceden la escala de pozo. Ejemplo de éstas, serían las correspondientes a exploración, prospección, distribución y procesamiento de energía. Para profundizar en esa dirección podrían ponderarse inversiones energéticas que se imputan a toda o parte de la formación, según la cantidad de pozos a desarrollar en el área explorada o prospectada.

En dirección contraria, la estimación presentada podría sobrestimar la inversión energética requerida. Ello sucedería si las actividades de transporte que actualmente demandan directamente grandes cantidades de combustible son reemplazadas por eventuales obras de infraestructura (por ejemplo, canalización de agua, vías ferroviarias, etc.). Lo mismo ocurriría si se redujeran las distancias de provisión de insumos o equipo. De hecho, se desarrolla la producción de agentes de sostén para las fracturas en el ámbito regional, lo cual evitaría el costo energético de transportarlos varios miles de $\mathrm{km}$ por mar y varios cientos de $\mathrm{km}$ por tierra.

\section{REFLEXIONES FINALES}

Los resultados de este trabajo evidencian incrementos en la demanda de energía fósil, tanto directos como indirectos, asociados con la "reprimarización" actual de la economía argentina. Para el caso de la expansión del agro-negocio y los consumos directos, se destacan los aumentos en la utilización de gasoil asociados con labores y transporte de granos. En cambio, el caso de la megaminería, la utilización de agroquímicos y el procesamiento de granos se asocian con consumos de combustibles fósiles indirectos (la electricidad utilizada proviene mayoritariamente de hidrocarburos). Una dificultad para determinar las transformaciones en la demanda de combustibles reside en las estadísticas disponibles. Por un lado, contabilizan los consumos de combustibles fósiles en actividades agrícolas, excluyendo el transporte de granos. Por otro, se integran consumos indirectos (tanto agroindustriales como mineros) en torno a la electricidad sin contemplar, entre otros aspectos, que la mayor parte de las usinas funciona con gas natural.

A su vez, al igual que lo estudiado para la extracción NC en Norteamérica, el rendimiento energético de la explotación de Vaca Muerta en Argentina, es inferior al tradicional. El menor retorno energético de la actividad se asocia por un lado, con una mayor inversión energética tanto directa (ej. combustible requerido para perforar, trasladar insumos, etc.) como indirecta (ej. combustible empleado para fabricar equipos e insumos). Por otro lado, la energía recuperada en los pozos NC podría ser inferior a la de los convencionales debido a que la "producción" decae con mayor velocidad que en los homólogos convencionales. En función de ello y del tipo de tecnología e intervención territorial que involucran, parece probable que para una misma cantidad de energía disponible los impactos negativos se amplifiquen.

Este trabajo es parte de una investigación en curso, motivo por el cual no permite realizar conclusiones definitivas. En cambio, los resultados alcanzados sugieren interrogantes adicionales. En esa dirección cabe reflexionar acerca de si el problema del autoabastecimiento energético en Argentina es de oferta o de demanda. Si fuera sólo de oferta, los avances derivados de la expropiación de Repsol permitirían resultados positivos al detener la depredación energética pero ¿qué ocurriría si la depredación energética es el resultado de la expansión del modelo extractivo? Reducir el des-balance energético con exclusiva atención de la oferta, implica continuar con las importaciones y/o intensificar las actividades extractivas internas de $\mathrm{HC}$, tanto convencionales como no convencionales. La fractura hidráulica, en ese marco, constituye un medio para atender una oferta que la reprimarización anticipa creciente. El estudio del fracking, mediante la focalización en la base biofísica de la economía, contribuye a revelar que la trama de conflictos distributivos en la Argentina actual, parece tejerse sobre la urdimbre común de los flujos energéticos.

\section{REFERENCIAS}

ALTOMONTE, H. CORREA, N. RIVAS, D. \& STUMPO, G.2011. La dinámica del consumo energético industrial en América Latina.

AUCOTT, M. \& MELILLO, J. 2013. A preliminary energy return on investment analysis of natural gas from the Marcellus shale. Journal of Industrial Ecology. 1-12.

BARRICK ARGENTINA. 2011. Resumen ejecutivo del Reporte de sustentabilidad 2010. (Fecha de acceso 15 de julio de 2014). Disponible en: http://barricklatam.com/barrick/site/artic/20131108/asoc file/20131108163319/reporte_barrick_argentina2010.pdf

BARRICK ARGENTINA. 2013. Resumen ejecutivo del Reporte de sustentabilidad 2012. (Fecha de acceso 27 de octubre de 2014). URL disponible en: http:/ / barricklatam.com/barrick/site/artic/20131108/asoc file/20131108163319/reporte_barrick_argentina2012.pdf

CALZADA, J. ROSSI, G. \& FERRER, N. 2015. El campo y la agroindustria argentina generaron 273 mil millones de dólares en exportaciones en 13 años. Informativo Semanal Nro. 1704. (Fecha de acceso 25 de Mayo de 2015). URL disponible en: http://www.bcr.com.ar/Pages/Publicaciones/infoboletinse manal.aspx?IdArticulo=1263 
Ambiente y Sostenibilidad 2015 (5): 97-109

Revista del Doctorado Interinstitucional en Ciencias Ambientales

ISSN: 2339-3122

Digital

CÁMARA DE LA INDUSTRIA ARGENTINA DE FERTILIZANTES Y AGROQUÍMICOS. 2014 Informe sobre consumo de fertilizantes en la campaña 2012/13. Fecha de acceso 23 de marzo de 2014. URL disponible on-line en: http://www.ciafa.org.ar/informes/Mercado/ConsumoFert ilizantes2013.pdf

CHARVAY, P. 2012. Los cambios en la producción ganadera en la posconvertibilidad. Voces en el Fénix 12: 22-29.

CHIAPPUSSI, F. 2013. Viaje al interior de Vaca Muerta, en Edición especial de National Geographics en español. Editorial Televisa, Editorial Atlántida. Páginas 22-35.

CLEVELAND, C. COSTANZA, R. HALL, C. \& KAUFMANN, R. K.1984. Energy US economy: a biophysical perspective. Science, 4665, 890-897.

CLEVELAND, C.J. \& O'CONNOR, P. 2011. Energy return on investment (EROI) of oil shale. Sustainability, 3(11), 2307-2322.

CONSEJO CIENTÍFICO INTERDISCIPLINARIO (CCI). 2009. Evaluación de la información científica vinculada al glifosato en su incidencia sobre la salud humana y el ambiente. (Fecha de acceso 12 de enero de 2013). Informe disponible on-line en: http:/ / www.reduas.fcm.unc.edu.ar/wpcontent/uploads/downloads/2013/06/2-CONSUMOAGROTOX-INFORME-2013.pdf

CORIA, M. 2005. Determinantes del consumo de combustibles líquidos en Argentina. Trabajo de investigación. Universidad Católica Argentina. (Fecha de acceso 15 de Julio de 2013). URL disponible en: http://200.16.86.50/digital/33/dt/aportespedagogicos/cor ia2-2.pdf.

DONATO, L. 2009. Estimación del consumo potencial de gasoil para las tareas agrícolas, transporte y secado de granos en el sector agropecuario. $X$ Congreso Argentino de Ingeniería Rural y II del Mercosur - Rosario (Sta.Fe, Argentina). Fecha de acceso 20 de Marzo de 2012. URL disponible en: http://inta.gob.ar/documentos/estimaciondel-consumo-potencial-de-gasoil-para-las-tareas-agricolastransporte-y-secado-de-granos-en-el-sectoragropecuario/at_multi_download/file/consumo3.pdf

DE-SOLMINIHAC, H. 2013. Energía, un insumo necesario para el crecimiento de la industria. In Seminario Minería y Energía. Santiago de Chile.

EL INVERSOR ENERGÉTICO \& MINERO. 2013. Neuquén puso en marcha el primer pozo no convencional bajo su gestión. (Fecha de acceso 12 de marzo de 2014). URL

disponible

en:

http:/ / www.inversorenergetico.com.ar/neuquen-puso-enmarcha-el-primer-pozo-convencional-bajo-su-gestion/

ENERGY INFORMATION ADMINISTRATION (EIA). 2011. World Shale Gas Resources: An Initial Assessment of 14 Regions Outside the United States. (Fecha de acceso 30 de Mayo de 2014). URL disponible en: http://www.eia.gov/oiaf/aeo/assumption/international.h tml

ENERGY INFORMATION ADMINISTRATION (EIA). 2014. Energy Units and Calculators Explained. Energy Conversion Calculators. Fecha de acceso 31 de Mayo de 2014). URL disponible en: http://www.eia.gov/energyexplained/index.cfm?page=ab out_energy_conversion_calculator

ENVIRONMENTAL PROTECTION AGENCY (EPA). 2011. The process of hydraulic fracturing. (Fecha de acceso 30 de Mayo de 2014). URL disponible en: www.epa.gov/ hydraulicfracturing/process.html

FERRANTE, S. B. \& GIULIANI, A. 2014. Fractura hidráulica: un abordaje no convencional para una extracción muy ortodoxa Vaca Muerta (Neuquén, Argentina) en perspectiva de economía ecológica. XIV Jornadas de Economía Crítica. Valladolid, 4 y 5 de septiembre.

FRECHERO, J. I. 2013. Extractivismo en la economía argentina. Categorías, etapas históricas y presente. Estudios Críticos Del Desarrollo, III (4), 45-82.

GARCÍA LINERA, A. 2012. Geopolítica de la Amazonía. Poder hacendal-patrimonial y acumulación capitalista. Vicepresidencia del Estado Plurinacional, Presidencia de la Asamblea Legislativa Plurinacional. La Paz, Bolivia.

GIAMPIETRO, M., \&SORMAN, A. 2012) Are energy statistics useful for making energy scenarios ? Energy, 37(1) 5-17.

GIULIANI, A. 2013. Gas y Petróleo en la economía neuquina. Educo, Neuquén, Argentina.

GREEN, M. B. 1987. "Energy in Pesticide Manufacture, Distribution and Use". En: ZR Helsel (ed.). Energy in Plant Nutrition and Pest Control. Elsevier: New York; en CCI 2009.

GUTIÉRREZ SCHMIDT, N. \& ALONSO, M. 2015. Informe anual 2014 no convencional la provincia de Neuquén. Petrotecnia, 48-60.

HELBLING, T. 2013. En alza: los altos precios y las nuevas 
tecnologías han propiciado un fuerte aumento de la producción de petróleo y gas en Estados Unidos que podría cambiar radicalmente los mercados energéticos mundiales. Finanzas y desarrollo 50(1), 34-37. Fondo Monetario Internacional y del Banco Mundial.

HOWARTH, R. W. SANTORO, R. \& INGRAFFEA, A. 2011. Methane and the greenhouse-gas footprint of natural gas from shale formations. Climatic Change, 106(4), 679-690.

KOZULJ, R. 2005. La crisis energética de la Argentina: orígenes y perspectivas. Fundación Bariloche IDEE paper, 7.

MANSILLA, D. 2011. Integración Energética y Recursos Naturales en América Latina. La revista del CCC. (Fecha de acceso 30 de Mayo de 2014). January/April 2011, n 11. URL disponible

en: http://www.centrocultural.coop/revista/articulo/212/.

MARTINEZ-ALIER, J. 1995. De la economía ecológica al ecologismo popular, Icaria/Nordan, Barcelona/ Montevideo.

MARCHESE, R. \&GOLATO, M. 2011. El consumo de combustible y energía en el transporte. Extensión 33. Revista de la Fac. de Cs. Exactas y Tecnología, Universidad Nacional de Tucumán, Argentina. (Fecha de acceso el 30/5/2014) URL disponible en http://www.herrera.unt.edu.ar/revistacet/ultimonro/nro3 3/pdf/n33ext02.pdf.

MERLINSKY, G. (ed). 2013. Cartografías del conflicto ambiental en Argentina. Buenos Aires, Argentina.

MINISTERIO DE ENERGÍA Y MINERÍA. 2014. Tablas dinámicas. (Fecha de acceso el 30/5/2014). URL disponible en:

http://energia3.mecon.gov.ar/contenidos/verpagina.php?i dpagina $=3299$

MOLTONI, L.\& DURO, S. 2014. Economía y Desarrollo Agroindustrial Incidencia de los herbicidas en los costos directos de la producción agrícola. Economía Y Desarrollo Regional, 3(1), 1-8.

MUDD, G. M. 2010. The Environmental sustainability of mining in Australia: key mega-trends and looming constraints. Resources Policy, 35(2), 98-115.

MUDD, G. M. 2007. Global trends in gold mining: Towards quantifying environmental and resource sustainability. Resources Policy, 32(1-2), 42-56.

MURPHY, D. J. \& HALL, C. 2010. Year in review--EROI or energy return on (energy) invested. Annals of the New York Academy of Sciences, 1185, 102-18.

MURPHY, D. HALL, CH. DALE, M. \&CLEVELAND, C. 2011. Order from Chaos: A preliminary protcol for determining the EROI of fuels. Sustainability, 3(11), 1888-1907.

ROULET, N. 2013. Gasoil: Aumento de costos en la campaña 2013/2014. Revista on-line EconoAgro. (Fecha de acceso 10 de Octubre de 2014) URL disponible en: http:/ / www.econoagro.com/632-gasoil-aumento-de-costosen-la-campana-2013/14

SABBATELLA, I. 2014. Neoliberalismo y naturaleza: la "comoditización" de los hidrocarburos en Argentina ( 19892001 ). Revista Iberoamericana de Economía Ecológica, 22, 101-116.

SECRETARIA DE MINERIA, MINISTERIO DE PLANIFICACIÓN FEDERAL, INVERSIÓN PÚBLICA Y SERVICIOS de la REPÚBLICA ARGENTINA. 2012. Minería en números. Informe publicado en la página web de la institución, (Fecha de acceso 12 de diciembre de 2014). URL disponible en: http://www.mineria.gob.ar/pdf/mineriaennumeros.pdf.

SELL, B., MURPHY, D., \& HALL, C. A. 2011. Energy return on energy invested for tight gas wells in the Appalachian Basin, United States of America. Sustainability, 3(10), 19862008.

SLIPAK, A. M.2013. ¿De qué hablamos cuando hablamos de reprimarización? Un aporte al debate sobre la discusión del modelo de desarrollo. Ponencia en las Jornadas de Economía Crítica, 29 al 31 de Agosto. Mendoza, Argentina.

SHIRATORI, J. 2012. Las empresas de ingeniería en el futuro petroquímico de la Argentina. Tecnología - Shale gas.Presentación en Jornadas del IPA. (Fecha de acceso el 2 de Septiembre de 2014). URL disponible en http://www.archivo.ipa.org.ar/images/27.06.2012JulioShir atori.pdf.

STERN, D. I.1997. Limits to substitution and irreversibility in production and consumption: A neoclassical interpretation of ecological economics. Ecological Economics, 21, 197-215.

TAILLANT, J. VALLS, M. D'ANGELO, M. HEADEN, C. ROELOFFS, A. 2013. Fracking Argentina:Informe Técnico y Legal Sobre la Fracturación Hidráulica en Argentina. Centro de Derechos Humanos y Ambiente \& ECOJURE. Córdoba, Argentina.

VALLEJO, M. C. 2006. La estructura biofísica de la economía ecuatoriana: el comercio exterior y los flujos ocultos del 
Ambiente y Sostenibilidad 2015 (5): 97-109

Revista del Doctorado Interinstitucional en Ciencias Ambientales

ISSN: 2339-3122

banano.Tesis . FLACSO - Sede Ecuador : Abya - Yala., Quito.

Digital

ISBN 9978-67-111-0. (Fecha de acceso el 5 de Octubre de

2012). URL disponible en: http:// biblioteca.ribei.org/58/.

YARITANI, H. \& MATSUSHIMA, J. 2014. Analysis of the Energy Balance of Shale Gas Development. Energies, 7(4), 2207-2227. 